\title{
Study of the macrostructure of steel (macroanalysis)
}

\author{
Cherney O.T. ${ }^{1}$, VaganovaO.I. ${ }^{2}$, Smirnova, Z.V. ${ }^{3}$, Bystrova, N.V. ${ }^{4}$ \\ ${ }^{1}$ Minin Nizhny Novgorod State Pedagogical University (Minin University), Nizhny Novgorod, Russian Federation, e-mail: \\ fiolet1975@mail.ru \\ ${ }^{2}$ Minin Nizhny Novgorod State Pedagogical University (Minin University), Nizhny Novgorod, Russian Federation, e-mail: \\ vaganova_o@mail.ru \\ ${ }^{3}$ Minin Nizhny Novgorod State Pedagogical University (Minin University), Nizhny Novgorod, Russian Federation, e-mail: \\ z.v.smirnova@mininuniver.ru \\ ${ }^{4}$ Minin Nizhny Novgorod State Pedagogical University (Minin University), Nizhny Novgorod, Russian Federation, e-mail: \\ bystrova_nv@mail.ru
}

\begin{abstract}
This study reveals the features of the macroanalysis of steel. The essence of the methods of macroanalysis is revealed. The basic concepts of conducting and preparing a study are considered, for more accurate information of the results. An example of studying the macrostructure of cast and deformed materials is given. An example of revealing a layer created by chemical heat treatment is considered.In the course of the study, a conclusion was made on the applied methods of macroanalysis of steel.
\end{abstract}

Key words: Macroanalysis, metal structure, steel, research.

\section{INTRODUCTION}

Macroanalysis - the study of the structure of the surfaces of workpieces and parts with the naked eye or at low magnification up to 30-50 times. All previous processing that the metal has undergone determines the macrostructure and nature of the fracture. Microscopic analysis of kinks is called fractography. The macrostructure can be studied directly on the surface of the part's forging casting or along a fracture. Most often, the macrostructure is studied on macrosections. A macrosection is a part of a part, forgings, castings, etc. specially prepared for macro-examination. The dimensions of macrosections are determined by the dimensions of the investigated part.

\section{RESEARCH}

The preparation of a macrosection is as follows: the part is cut into 2 parts. One surface of the part is cleaned on a machine and polished with various numbers of metallographic abrasive paper, sequentially moving from a coarse number to a thin one; each time the macrosection is turned $90^{\circ}$. Grinding in one direction is carried out until the risks from the previous paper number disappear. Grinding can be done manually or on a special machine.

After grinding to reveal the macrostructure, the thin sections are subjected to deep etching with chemical reagents (solutions and a mixture of acids and alkalis), which are selected depending on the composition of the alloy and the purpose of the study [1].
Macroanalysis allows you to determine the quality of the metal, to establish deviations from the technological mode of processing, to select those areas of the studied part that need to be subjected to more detailed further research. Control of the macrostructure and fractures of steel, regulation of the macrostructure and permissible macrodefects of carbon and alloy structural and tool steels are provided for in GOST 10243-75 "Steel. Test methods and evaluation of macrostructure."[2].

Macroanalysis allows you to establish:

1) the structure of the metal or alloy in cast parts or ingots (granular, dendritic), the presence and distribution of crystallization zones, shrinkage looseness, bubbles, cracks, etc.;

2) chemical heterogeneity obtained during crystallization. Sulfur, phosphorus and carbon in the process of crystallization tend to be unevenly distributed over the section of the ingot, which greatly affects the properties of its individual sections. This phenomenon is called liquation, not eliminated by subsequent processing;

3 ) the structure of the metal after hot forming (rolling, stamping, forging, etc.). After processing by pressure, the internal structure of metals and alloys turns out to be denser than after casting, since in the process of hot processing by pressure they are partially destroyed porosity, looseness, cracks, etc.

In the process of pressure treatment, structural components, as well as non-metallic inclusions, are crushed and stretched along the direction of deformation, forming longitudinal fibrillation. The mechanical properties of the metal are different depending on the direction of the fiber. The impact strength across the fiber is 3-4 times higher than along the fiber. For parts operating at increased specific loads (crankshafts, valves, gears, etc.), it is necessary that the fibers are parallel to the contour of the part. This is achieved by the correct choice of pressure treatment methods;

4) heterogeneity of composition and structure created by thermal or chemical-thermal treatment. Parts that must have a high surface hardness while maintaining a tough, ductile core are usually surface hardened or chemically heat treated, i.e. surface saturation with carbon (carburization), nitrogen (nitriding) or combined saturation with carbon and nitrogen (cyanidation) and etc. with or without subsequent heat treatment. Macroanalysis 
determines the depth and nature of the distribution of the hardened, cemented, nitrided, cyanide layer;

5) the nature of the fracture of the metal or alloy. A fracture can be used to determine the nature of the fracture: ductile, brittle or caused by fatigue [3].

Fracture - the surface of a specimen or part completely destroyed along a main crack as a result of mechanical single or repeated loading of any kind: tension, compression, bending, torsion, shear, as well as with various combinations of loads and additional influence of various media, fracture with the formation of a fracture can occur under the action of internal stresses (thermal, structural, etc.); is a valuable characteristic of the quality of the material, and also reflects the local conditions of the processes of deformation and destruction.

Fibrous - sometimes called viscous, more precisely, ductile fracture of metals; characterized by an almost complete absence of crystal facets on the surface; the structural features are due to the deformation of the grains, stretched out into "fibers". Most often accompanied by clear signs of deformation near the fracture. Contrasted with crystalline fracture.

Stone-like - fracture, sometimes called tuberous; characterized by a coarse-grained, lumpy structure; grains on the surface do not have an obvious metallic luster, they appear "melted". Occurs in overheated and then heat treated steel. With a weak development of the defect in the fracture, only separate areas or grains of a stone-like structure are observed.

Crystalline - a brittle fracture of materials with a crystalline structure; characterized by the presence of shiny, flat areas (facets) on the surface; arises under conditions of destruction with low plastic deformation; the degree of plastic deformation preceding fracture is usually also small; however, crystalline fracture can also occur after significant plastic deformation. Distinguish between coarse-crystalline and fine-crystalline fractures, respectively, with large and small facets on the surface [4].

Intergranular - fracture at grain boundaries. To characterize the fracture, the more correct term should be considered grainy

Naphthalene - fracture is characterized by a coarsegrained structure in the presence of smooth, shiny, nonmetallic facets on the surface: it is found in tool steels, mainly high-speed steels, with the wrong technology of hot mechanical and heat treatment.

Brittle - fracture, not accompanied by noticeable plastic deformation; in metals it has, as a rule, a crystalline structure.

Porcelain-like - fine-crystalline, smooth fracture of metals, in appearance resembling the fracture surface of porcelain; used to characterize the appearance of both fatigue and single fractures.

Floken - is a crack that looks like a white spot with a larger crystalline structure in a fracture than the general background of the fracture. Shades of white and the degree of crystallinity can vary depending on the steel grade and the conditions for the formation of flocs.

Slate - a fracture with a tree-like, layered structure; the layers are in the form of threads of different lengths almost parallel to each other, ending in transverse ledges; observed in forged or rolled metal.
Glossy - a fracture with a smooth, as if ground-in surface; occurs in the zone of actual fatigue crack propagation, most often under cyclic alternating loads; can also be observed under cyclic tension with significant static loading, for example, in case of fatigue failure of overtightened studs

\section{RESULT AND DISCUSSION}

Having studied the macrostructure and fractures of steel, we conducted a study to study the macrostructure of cast and deformed materials. Revealing the layer created by chemical thermal treatment.

The surface of the prepared macrosection is etched with a $15 \%$ aqueous solution of nitric acid for $10-15 \mathrm{~s}$. After etching, the section is wiped with a cotton swab soaked in alcohol. Visually (roughly) the depth of the layer obtained as a result of chemical-thermal treatment is determined.

Polished samples are wiped with a special solution for the purpose of degreasing and etched with an appropriate reagent, depending on the task.

Macrosection of calm cast steel deoxidized with manganese, silicon and aluminum. When the metal crystallizes in a casting mold, branched tree-like dendrite crystals are often formed in the (mold). Dendritic macrostructure is a casting feature. Crystallization begins at the walls of the mold and spreads deep into the liquid mass of the metal.

If the technology for producing an ingot (casting) is observed, the macrostructure of casting in most cases has three characteristic zones with different structures: 1 zone of fine-grained dense crust (small equilibrium crystals). The formation of this zone is influenced by a high cooling rate due to the large temperature drop between the cold wall of the mold and the molten metal; 2 - zone of columnar crystals that grow in the direction of heat removal and have the same orientation; 3 - zone of equiaxed crystals, the dendrites of which are oriented in different directions and can be of different sizes. Figure 1. The volume of the metal located between the dendrites crystallizes in the last turn, therefore, insoluble impurities, non-metallic inclusions are mainly concentrated in the central part of the ingot. In addition, due to a decrease in the volume of the metal during the transition from a liquid to a solid state, microcavities and pores are concentrated in these layers. During subsequent rolling, these macrodefects must be welded.

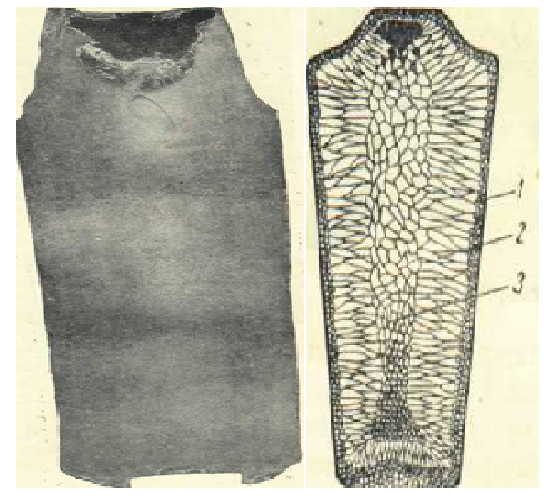

Figure 1: Macrostructure of a longitudinal section of a $700 \mathrm{~kg}$ steel ingot 
A characteristic defect of a calm steel ingot is a shrinkage shell. Various methods are used to achieve the location of the shrinkage cavity in the upper part of the ingot, which in large-tonnage ingots (10 and more tons) is cut off before rolling, and high-quality metal is used for rolling. The yield of good rolled products from calm steel ingots is $85-90 \%$ [5].

Macrosection of a boiling steel ingot Figure 2. This steel is deoxidized by manganese and differs in chemical composition from quiet steel by a very low silicon content ( $\mathrm{Si}<0.7 \%$ ). Compared to calm steel, boiling steel has high ductility and deforms well in the cold state. A characteristic feature of the macrostructure of boiling steel is a large number of gas bubbles, which are formed as a result of carbon deoxidation, and the absence of a shrinkage cavity in the ingot. Gas bubbles, the surface of which is not contaminated with slag and non-metallic inclusions, are welded during hot rolling. The yield of good rolled products from boiling steel ingots is $95 \%$ or more.

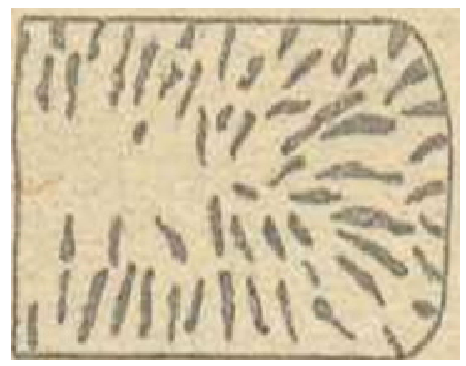

Figure 2: Macrosection of a boiling steel ingot (honeycomb bubbles)

Sulfur print on photographic paper from a cast steel sample. The nature of the distribution of non-metallic inclusions in steel can be judged by the sulfur imprint on photographic paper [6]. The print provides a qualitative assessment of the inhomogeneity of the sulfur distribution in steel. Uneven distribution of individual elements leads to the formation of new defects and to a decrease in the mechanical properties of finished products: for example, sulfur segregation can cause red brittleness (cracking during hot plastic deformation) Figure 3.

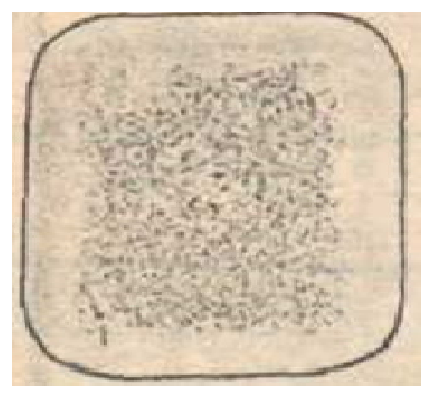

Figure 3: Elimination

Casting with continuous transcrystallization zone. In small ingots and castings obtained in metal molds, especially when pouring overheated metal, the columnar crystal zone can occupy the entire volume of the ingot (casting), right up to the center. At the junction of columnar crystals, due to micro- and macrovoids, the metal has a reduced strength ("weakness"), and during subsequent pressure treatment in these areas, cracks may form. Figure 4.

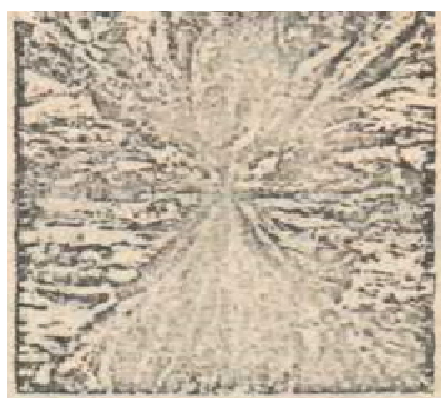

Figure 4: Casting with continuous transcrystallization

Macrosection with fibrous macrostructure. In the process of hot plastic deformation, primary dendrites and nonmetallic inclusions of interdendritic spaces stretch in the direction of deformation and acquire a fibrous structure that repeats the configuration of the part. The mechanical properties of the deformed metal depend on the direction of the fibers (anisotropy of properties) [7]. By forging or punching, the aim is to create such an arrangement of the fibers so that it is perpendicular to the effective stresses in Figure 5 .

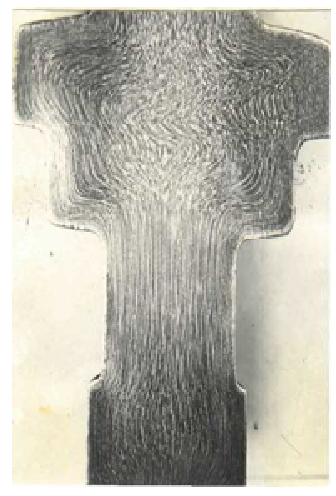

Figure 5: Macrosection of deformed metal (fibrous structure)

\section{CONCLUSION}

Thus, in the process of studying the macrostructure of cast and deformed materials, we examined the samples and identified methods for testing and evaluating the macrostructure.

Microscopic analysis consists in examining the structure of metals using a microscope. With the help of microanalysis, the phase composition and structural components of the metal or alloy are studied; size, shape and orientation of grains; the presence of defects that reduce the quality of the metal (inclusions, microcracks, etc.). The result of the microanalysis is a conclusion on the nature of the material processing, on the compliance of the structure with the technical conditions, on the violation of technology. 


\section{REFERENCES}

1. Arzamasov B.N., Cherepakhin A.A. Materials science: textbook for universities (bachelor's degree). M.: Academy, 2013.

2. LakhtinYu.M., Leontyeva V.P. Materials science: textbook for universities.3rd ed. stereotype. M.: EKOLIT, 2011.

3. Pachurin G.V., Shevchenko S.M., Mukhina M.V., Kutepova L.I., Smirnova J.V. The factor of structure and mechanical properties in the production of critical fixing hardware 38XA //

Tribology in Industry. 2016. T. 38.No. 3.P. 385-391. https://doi.org/10.5831/HMJ.2016.38.2.385

4. Cherney O.T., Zotova V.A., Permovsky A.A., Smirnova Z.V., Vaganova O.I. Hydrogenous phase concentration: impact on the structural condition and fracture mechanics of technically pure titanium after different conditions of hydrogenation // International Journal of Innovative Technology and Exploring Engineering. 2019.Vol. 8.No. 6.P. 535-539.

5. Zh.V. Smirnova, O.T. Cherney. Construction material technology textbook / Minin University. Nizhny Novgorod, 2019.

6. Y. Pratapa Reddy, Dr. K. L Narayana, Dr. M. Kedar Mallik. Electro-chemical behavior of different metals in Sodium Chloride solution. // International Journal of Emerging Trends in Engineering Research, 7 (11), November 2019, 71 Volume 7, No. November 11, 2019.

7. EkoJulianto, Waluyo Adi Siswanto, Marwan Effendy Characteristics of Temperature changes and Stress of Float Glass under Heat Radiation // International Journal of Emerging Trends in Engineering Research, Volume 7, No. September 9, 2019.

https://doi.org/10.30534/ijeter/2019/03792019 